Nichtinvasive Bestimmung der Gewebeperfusion in der Vorderarmmuskulatur von narkotisierten Patienten mit Hilfe einer neuen Ultaschalldoppler-Methode

\author{
Bührer, A ; Moser, U ; Pasch, T
}

DOI: https://doi.org/10.1515/bmte.1996.41.s1.174

Posted at the Zurich Open Repository and Archive, University of Zurich ZORA URL: https://doi.org/10.5167/uzh-154363

Journal Article

Published Version

Originally published at:

Bührer, A; Moser, U; Pasch, T (1996). Nichtinvasive Bestimmung der Gewebeperfusion in der Vorderarmmuskulatur von narkotisierten Patienten mit Hilfe einer neuen Ultaschalldoppler-Methode. Biomedizinische Technik. Biomedical engineering, 41(s1):174-175.

DOI: https://doi.org/10.1515/bmte.1996.41.s1.174 


\title{
Nichtinvasive Bestimmung der Gewebeperfusion in der Vorderarmmuskulatur von narkotisierten Patienten mit Hilfe einer neuen Ultaschalldoppler-Methode
}

\author{
Bührer A. ${ }^{1.2}$, Moser U. ${ }^{1}$, Pasch T. ${ }^{2}$ \\ 'Institut für Biomedizinische Technik und Medizinische Informatik, \\ ETH und Universität Zürich, CH-8092 Zürich, Schweiz \\ 2 Institut für Anästhesiologie, Universitätsspital Zürich, CH-8091 Zürich, Schweiz
}

\section{DIE PROBLEMSTELLUNG}

Die Gewebeperfusion ist das durch die terminale Strombahn fliessende Blutvolumen pro Zeit und pro Gewebegewicht $(\mathrm{ml} / \mathrm{min} / 100 \mathrm{~g})$ oder pro Gewebevolumen $(\mathrm{ml} / \mathrm{min} / 100 \mathrm{ml})$. Die terminale Strombahn besteht aus Arteriolen, terminalen Arteriolen, Kapillaren, postkapillären Venolen, Venolen und arteriovenösen Anastomosen. Sie ist von grosser physiologischer und pathophysiologischer Bedeutung. Einerseits tragen die Arteriolen - auch Widerstandsgefässe genannt - den weitaus grössten Teil zum totalen Gefässwiderstand bei. Andererseits finden in den Kapillaren und den postkapillären Venolen die Austauschvorgänge zwischen Blut und interstitieller Flüssigkeit statt. Diese sind für die Versorgung der einzelnen Zellen von grosser Wichtigkeit.

Trotzdem gibt es noch keine wirklich befriedigende Messmethode zur Bestimmung der Gewebeperfusion, um anästhesiologische oder intensivmedizinische Fragestellungen beantworten zu können. Die ideale Messmethode sollte einfach zu handhaben, nicht-invasiv, kontinuierlich und genau sein. Unter den heute bekannten Messmethoden ist die gepulste Ultraschall-Dopplertechnik eine der geeignetsten. Dies obwohl sie wie alle Dopplerverfahren nur semiquantitativ ist. In dieser Arbeit wird ein neu entwickeltes gepulstes Ultraschall-Dopplersystem zur Erfassung der Gewebeperfusion in der Anästhesiologie vorgestellt sowie erste Resultate aus einer grösseren Patientenstudie präsentiert.

\section{DAS ULTRASCHALL-DOPPLERSYSTEM}

Das Konzept des neu entwickelten Ultraschall-Dopplersystems (USDS) ist im wesentliche durch die langsamen Fliessgeschwindigkeiten der Erythrocyten, das schwache Dopplersignal (tiefes Signal-zu-Rauschverhältnis) und das Bedürfnis, über ein echtzeitfähiges System zu verfügen, bestimmt. Das System besteht aus vier Hauptkomponenten, nämlich einem $4 \times 4$ Arraytransducer, dem eigentlichen Ultraschall-Dopplermodul, einer leistungsfähigen Signalprozessorkarte (DSP-Karte) und einem IBM-kompatiblen Personalcomputer (PC).

Aus technischen Gründen wurde ein $4 \times 4$ Arraytransducer mit runden $2 \mathrm{~mm}$ Elementen gewählt. Der Transducer ist unfokussiert, so dass die laterale Ausdehnung des Schallfelds im interessierenden Tiefenbereich (bis $20 \mathrm{~mm}$ ) etwa der Ausdehnung der Transducerelemente
$(8 \times 8 \mathrm{~mm})$ entspricht. Die empfangenen Echosignale werden noch im Transducergehäuse vorverstärkt. Der Transducer wurde von der Firma Medizintechnik Basler AG (MTB), Zürich, Schweiz nach unseren Angaben hergestellt.

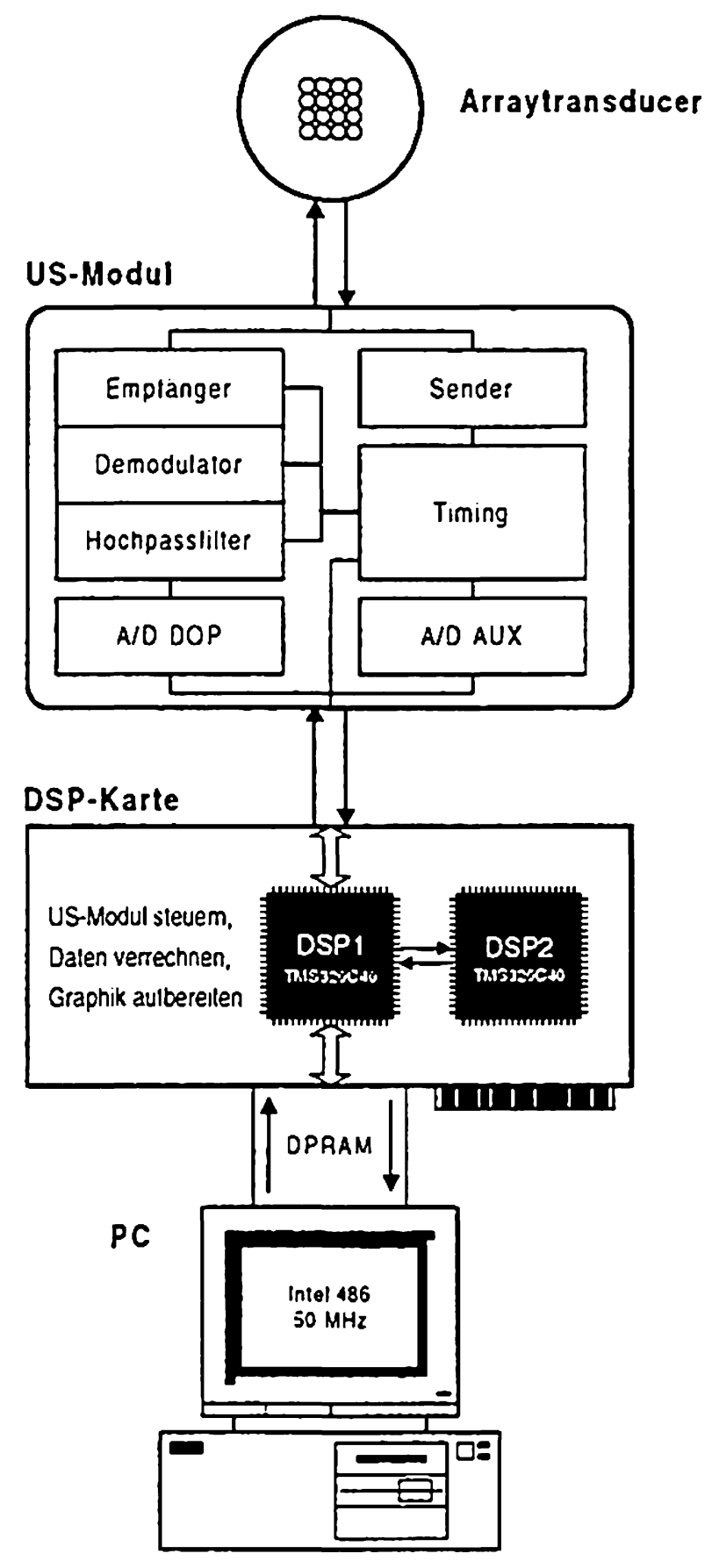

Das Ultraschall-Dopplersystem besteht alls vier Hauptkomponenten, dem Arraytransducer, dem eigentlichen Ultraschall-Dopplermodul (US-Modul), einer Signalprozessorkarte (DSP-Karte) und einem PC.

Das Ultraschall-Dopplermodul ist mit vier gepulsten $15 \mathrm{MHz}$ Sendern und zwei Empfangskanälen ausgerüstet. Für Messtiefen bis $2 \mathrm{~cm}$ wird bei einer Sendefrequenz von $15 \mathrm{MHz}$ ein optimales Signal-zu-Rauschverhältnis erreicht. Ein weiterer Vorteil der hohen Sendefrequenz liegt 
in der entsprechend höheren Dopplerfrequenzverschiebung. Dies ist von Bedeutung, weil die Fliessgeschwindigkeiten der Erythrocyten in den Kapillaren mit $0-2.5$ $\mathrm{mm} / \mathrm{s}$ sehr klein sind (mittlere Geschwindigkeit in der Aorta: $200 \mathrm{~mm} / \mathrm{s}$ ). So erzeugt ein sich mit $0.5 \mathrm{~mm} / \mathrm{s}$ in Transducerrichtung bewegender Erythrocyt bei einer Sendefrequenz von $15 \mathrm{MHz}$ eine Dopplerfrequenzverschiebung von lediglich $10 \mathrm{~Hz}$. Die Sendepulslänge und das Empfangstor betragen je $1.07 \mu \mathrm{s}$, so dass die Messschicht etwa $1 \mathrm{~mm}$ dick wird. Das Empfangssignal jedes Kanals wird orthogonal demoduliert, hochpassgefiltert (Unterdrückung der Stationärechos) und mit 14 Bit Auflösung digitalisiert, so dass ein komplexzahliger Datenstrom resultiert. Zusätzlich verfügt das Dopplermodul über vier Hilfskanäle (AUX), welche gleichzeitig weitere Signale wie EKG, Laserdopplerflux etc. registrieren können. Die Doppler- und die Hilfskanaldaten werden seriell an die Signalprozessor-Karte weitergeleitet.

Die Signalprozessor-Karte (DSP-Karte) ist eine PC-Einsteckkarte des Typs DPC/C40D der Firma Loughborough Sound Images Limited (LSI), England. Sie ist mit zwei Hochleistungsprozessoren (DSP1 und DSP2) des Typs TMS320C40 (Texas Instruments, USA) bestückt. Die DSP-Karte kontrolliert das Ultraschall-Dopplermodul, berechnet Signalleistungsspektren sowie die Gewebeperfusion und bereitet die Resultate für die graphischen Darstellung auf dem PC vor.

Der IBM-kompatible Personalcomputer (Intel 80486, $50 \mathrm{MHz}$ ) dient im wesentlichen als Benutzerschnittstelle, welche unter anderem die Daten in Echtzeit visualisiert. Die Signalleistungsspektren werden farbcodiert dargestellt. Die Gewebeperfusionswerte werden den Spektren ïberlagert präsentiert. Im weiteren archiviert der PC die anfallenden Resultate. Das PC-Programm ist in "Visual $\mathrm{C}++$ " unter "Windows 3.11 " codiert.

\section{MESSUNGEN AN PATIENTEN}

\section{Protokoll}

Um das USDS auf seine Tauglichkeit zu überprüfen, wurden insgesamt an 35 Patienten Messungen durchgeführt. Zur Studie zugelassen waren Patienten der ASA Klassen 1 und 2, welche in Allgemeinanästhesie operiert wurden. Als Anästhesiegas wurde in altemierender Reihenfolge Enfluran (E) oder Isofluran (I) verwendet. Eine erste dreiminütige Messung wurde am wachen Patienten durchgeführt. Eine zweite folgte unmittelbar nach Intubation bei $100 \%$ Sauerstoffgabe noch ohne Anästhesiegasgabe (Messdauer $1 \mathrm{~min}$ ). In den nachfolgenden Messungen (Messdauer $3 \mathrm{~min}$ ) wurde zuerst die endtidale Anästhesiegaskonzentration und anschliessend das Beatmungsmuster variiert. Zum Schluss wurde ein postokklusiver reaktiver Hyperämieversuch durchgeführt. Das Ende der dreiminütigen Ischämiephase dieses Versuches wurde dabei als biologischer Nullwert verwendet.

Die Muskelperfusion wurde mit Hilfe des USDS in der ulnaren Beugemuskulatur des rechten Vorderarmes in einer Tiefe von $10 \mathrm{~mm}$ bestimmt (Pulsrepetitionsfrequenz: $13.7 \mathrm{kHz}$, Beobachtungsdauer: $150 \mathrm{~ms}$, Geschwindigkeitsauflösung: $0.33 \mathrm{~mm} / \mathrm{s}$ ). Zusätzlich wurde die Haut- perfusion mit Laserdopplerflowmetrie mit $13.4 \mathrm{~Hz}$ über die Hilfskanäle des USDS aufgezeichnet. Vor und nach jeder Messung wurden zudem die Herzfrequenz (HR), der systemische Blutdruck (BD), die arterielle Sauerstoffsättigung, die endexpiratorische Kohlendioxidkonzentration sowie das Atemzugvolumen protokolliert.

\section{Resultate}

Insgesamt wurden während 1000 Minuten erfolgreich Perfusionsdaten aufgezeichnet. Einer der 35 Versuche musste wegen Störfrequenzen unbekannter Herkunft (anderes elektrisches Gerät ?) verworfen werden.

Die gefundenen Muskelperfusionswerte stimmen mit den Werten aus der Literatur überein und sind medizinisch relevant. So sank die mediane normierte Muskelperfusion unter Enfluran mehr als unter Isofluran ab (E: $1 \rightarrow 0.58$, I: $1 \rightarrow 0.83 ; p \leq 0.02$ ). Ebenfalls den Erwartungen entsprechend verhielten sich die Herzfrequenz und der Blutdruck. So stieg die mediane HR im Isoflurankollektiv von 78 auf 89 Schläge / min an. Die HR im Enflurankollektiv blieb dagegen praktisch gleich $(72 \rightarrow 69)$. Der Blutdruck sank unter Enfluran auf deutlich tiefere Werte als unter Isofluran (syst., mittl., diast. BD [mm Hg]; E: 119/90/76 $\rightarrow$ 95/72/62; I: 130/97/84 $\rightarrow$ 110/85/72). Zwischen Perfusion und HR resp. BD wurde ein ausgeprägter gleichsinniger Zusammenhang nachgewiesen. Zwischen der Muskelperfusion und der Hautperfusion konnte dagegen kein einfacher Zusammenhang gefunden werden.

\section{SCHLUSSFOLGERUNGEN}

Diese erste Patientenstudie hat gezeigt, dass das hier entwickelte Ultraschall-Dopplersystem geeignet ist, anästhesiologische und intensivmedizinische Fragestellungen $z \mathrm{u}$ untersuchen. Um allerdings den definitiven Stellenwert dieser Methode festlegen zu können, sind noch weitere Messungen am Tier und am Menschen notwendig. Besonders interessant wird es sein, an parenchymatösen Organen oder am Gehim zu messen.

\section{LITERATUR}

BASLER, S. Nichtinvasive Erfassung der Gewebsdurchblutung mittels Ultraschall. Dissertation Nr. 8796 der Eidgenössischen Technischen Hochschule Zürich; 1989

BÜHRER, A. Erfassung der Gewebeperfusion in der Anästhesiologie mit Hilfe eines Ultraschall-Dopplersystems. Dissertation Nr. 11700 der Eidgenössischen Technischen Hochschule Zürich; 1996

BÜHRER, A., MOSER, U. T., SCHUMACHER, P. M., PASCH, T., ANLIKER, M. Subtraction procedure for the registration of tissue perfusion with Doppler ultrasound. Ultrasound Med. Biol. 22: Nr. 4; 1996

DYMLING, S. O., PERSSON, H. W., HERTZ C. H. Measurement of blood perfusion in tissue using Doppler ultrasound. Ultrasound Med. Biol. 17: 433 - 444; 1991 